\title{
The Effect of Scientific Collaboration on CSCW Research: A Scientometric Study
}

\author{
António Correia $^{* 1,2}$, Shoaib Jameel ${ }^{2}$, Daniel Schneider ${ }^{3}$, Benjamim Fonseca ${ }^{1}$, and Hugo Paredes ${ }^{1}$ \\ ${ }^{1}$ INESC TEC and University of Trás-os-Montes e Alto Douro, UTAD, Quinta de Prados, Apartado 1013, Vila Real, Portugal \\ ${ }^{2}$ University of Kent, School of Computing, Medway Campus, UK \\ ${ }^{3}$ Tércio Pacitti Institute of Computer Applications and Research (NCE), Federal University of Rio de Janeiro, Brazil \\ ${ }^{*}$ Corresponding author. Email address: antonio.g.correia@inesctec.pt
}

\begin{abstract}
The structure and evolution of a scientific research community can be quantitatively assessed taking into account the interactions between scientific agents dispersed geographically. In the recent years, CSCW has stabilized as a cross-disciplinary field suffering significant changes in its core structure, and there is limited understanding about the factors influencing the nature and progress of collaborative computing research. In this paper, we measure the correlation between a set of features related to the influence of collaboration types on the number of citations as well as the geographical distribution of the accumulated contribution to the CSCW literature. Overall, our work can represent a starting point to demonstrate how the study of scientific collaboration can partly explain the variations in the number of citations, frequency of papers, and topics addressed.
\end{abstract}

Keywords-bibliometrics; citation analysis; collaboration types; collaborative scientific research; CSCW; geographical distribution; scientific collaboration; scientometrics

\section{INTRODUCTION}

Most of the scientific work involves a social construction process that requires a certain degree of cooperation [1]. Moreover, the considerable growth of literature offers unparalleled possibilities for measuring science from largescale data sources [2]. Committed to inform the development of new systems and architectures intended to support technologically enabled or enhanced collaboration, the field of Computer Supported Cooperative Work (CSCW) has presented a vast set of theoretical constructs to characterize the evolving cooperative work arrangements and technology-driven waves that shape digitally mediated communication and collaboration [3]. This is validated through the large volume of papers annually published by CSCW researchers. A simple search in the ACM Digital Library ${ }^{1}$ denotes that the field has apparently quadrupled the number of publications since the beginning of the century. However, there is a significant amount of research (e.g., $[4,6])$ that point to a manifested lack of reflection about the component factors of collaboration within the field of CSCW coupled with a need for demonstrating its impact within and beyond the scientific community [7].

Although recent efforts have been made to describe some technical aspects of collaborative systems (e.g., synchronicity) from literature (e.g., [8, 9]), many challenges arise when

\footnotetext{
${ }^{1}$ https://dl.acm.org/
}

considering the complexity of examining individual and collaboration outcomes whitin a highly multidisciplinary and polymorphic field of research. To the best of our knowledge little is known about how attributes and types of research collaboration affect researchers' impact in the field of CSCW. Scientific agencies, students, faculty members, publishers, conference organizers, research institutes and laboratories, and the general public can be informed by such kind of quantitative examinations to understand the nature of a field and its evolution over time, including structural gaps and trends, scientific networks, and sociological phenomena $[10,11]$.

The value proposition of this study is that a broad understanding of the factors influencing the structure and impact of scientific collaboration among CSCW authors can better explain the mechanisms responsible for scientific discovery, following Fortunato and colleagues [2]. Thus, this work presents scientometric data as formative instruments and integral units of the "science of measuring" [14] to analyze the CSCW literature between 2001 and 2015. The paper aims to reflect on the impact of the published papers in the first 15 years of this century by assessing authorship and citation data, collaboration patterns, and research topics. In the course of these scientometric examination activities, we focus on the social dimension of academic collaboration, including the past interactions between authors in the field of CSCW.

The paper is outlined as follows. We first discuss earlier work related to our study, giving attention to past bibliometric studies on collaborative scientific research and CSCW (Section 2 ). We then detail the data, design, and methods for our study (Section 3). Next, we present our findings and provide our interpretations of these results (Section 4). Last but not least, we conclude with a summary of our findings, their limitations, implications, and thoughts for future research (Section 5).

\section{RELATED WORK}

Scientometrics [15] can be described as a scientific specialty concerned with the quantitative study of scientific and technological domains as measurable multidimensional constructs [16]. Some of the main themes addressed in this field emphasize the study of knowledge representations as well as cognitive and socio-organizational processes in terms of structure, growth, and impact. Van Raan [17] goes even further by identifying the interest on aspects related with social factors 
and the appropriate development of information and software technologies for scientific data gathering, examination and dissemination based on bibliometric indicators such as citations, co-authorship networks, demographics, keywords, and alternative metrics (e.g., downloads, readers, and social mentions) [7]. The foundations for the field have been discussed in the literature since the late 1960s (see Mingers and Leydesdorff [18] for a review on the history of scientometrics).

\section{A. Collaborative Scientific Research}

The recent advances in IT and the growing complexity, cost and scale of scientific research have led collaborative scientific research to become a critical element in several communities and disciplines [19]. Scientific collaboration involves a dynamic, co-evolving multiscale network of actors and knowledge representations guided by complex interactions between social sctructures [2]. Many patterns have been unveiled through the study of collaboration and citation networks. Wuchty et al. [19] observed that co-authored papers tend to receive more citations. Moreover, the scientific impact of an article is greatly influenced by the increased number of authors (i.e., size of research teams) [20]. At a macro level, collaboration can be also positively correlated with academic quality, and this is one of the main factors by which scientific collaboration is growing in terms of incidence and size [20]. Other factors include the limited amount of time and resources (e.g., funding, knowledge, and equipment) [21] as well as the need to challenge disciplinary boundaries [22], enhance legitimacy and deal with high levels of complexity [23], and achieve novel combinations of ideas [24].

There is also a significant amount of studies on aspects such as persistence and degree of transdisciplinarity between authors [25], cohesion and closeness of authors as measured by co-authorship networks [26], authors' scientific impact [27], and role of social relations in the writing of scientific papers (including the social dimension of citations) [28]. Considering the drawbacks and negative aspects of scientific collaboration, some researchers have found practical barriers related with communication among members, resulting in inter-personal conflicts and additional effort, resources, and time investment [30]. Past findings also denoted a negative impact of long-term collaboration and team size on group's performance and quality of published papers [21]. Further examinations showed that collaborative interactions between researchers can be also negatively impacted by factors such as distance, cultural divides, language, lack of funding, and task overload. In recent years, there has been increasing interest in crowdsourcing research as a means to overcome such limitations [29, 41].

\section{B. CSCW and Scientific Practices}

As far as we know, the first known study assessing the quantitative aspects of CSCW literature in this millenium was presented by Holsapple and Luo in 2003 [10]. The authors analyzed a total of 19,271 citations to track the impact of CSCW reseach from 1992 to 1999. Their findings revealed that the number of citations remained stable until the end of the last century. A co-authorship network analysis [4] demonstrated that $\mathrm{CSCW}$ authors maintained a great percentage of collaborative interactions with researchers from other fields between 1999 and 2003. However, physical proximity represented a critical factor in establishing collaborations between HCI and CSCW researchers. The authors went even further by applying co-authorship networks and citation data analysis on the ACM CSCW conference proceedings. Meanwhile, Jacovi and colleagues [11] mapped the field through a citation graph analysis of the ACM CSCW conference series between 1986 and 2004, identifying a small level of papers without citations. Extrapolating to the study of demographics in the ACM CSCW conference (1986-2002) [32], results revealed a high number of contributions by academics from USA (70-90\%) compared with European researchers $(10-30 \%)$. This study also denoted a great focus on group issues and design aspects of system prototypes and architectures as well as an increasing number of publications on ethnography and experimental approaches.

A careful analysis of earlier studies led us to other quantitative examination of the ACM CSCW conference series (1998-2004) [33]. The authors identified a stable portion of design and evaluation studies, a high number of descriptive studies, a constant growth in papers testing hypotheses about group dynamics and collaboration issues through experiments, and a decline of non-empirical papers. Moreover, Keegan and co-workers [6] used scientometrics and social network analysis to identify that the impact of $\mathrm{CSCW}$ authors and papers is strongly influenced by their structural position within inner collaboration and citation networks. A quantitative analysis of CSCW research was conducted by Correia and colleagues [7]. This study identified a significant number of scientometric studies on CHI conference and related venues (e.g., [35]). Mubin and co-workers [36] argued that such studies have largely applied techniques that range from author and trend analysis to co-authorship networks, institutional-level analysis, topic modeling, and citation analysis.

\section{METHOD}

Scientometrics has proven to be an effective approach for assessing scientific outputs and their complex structures [7]. According to $\mathrm{Bu}$ and co-authors [21], ten years is a sufficient period for researchers to set up and develop their research. This study relies on a sample of CSCW research of which only 1713 papers published between 2001 and 2015 were selected for examination from four devoted outlets: ACM Conference on Computer-Supported Cooperative Work and Social Computing (ACM CSCW), ACM International Conference on Supporting Group Work (GROUP), European Conference on ComputerSupported Cooperative Work (ECSCW), and Computer Supported Cooperative Work: The Journal of Collaborative Computing and Work Practices (JCSCW).

Our analysis was limited to the venues with more than a quarter century of existence in the field of CSCW. Such venues were also chosen due to their specificity $[4,10]$ and because their scientific committees and editorial boards comprise some of the most cited researchers in CSCW, "providing a representative (although limited) sample of the work published in this century" [7]. It is also noteworthy that these venues have been used as sources for other scientometric studies (e.g., [4, 6, $10,11])$. Excluded records include plenary sessions, panels, posters, keynotes, extended abstracts, editorials, and tables of 
contents. At this stage, we excluded regional conferences such as British HCI, OzCHI, NordiCHI, and ChineseCSCW. Nonetheless, we are aware that our research should be validated by a larger sample size. The picture is thus still incomplete and further work needs to be done to characterize other venues focused on the research and development of human-computer interfaces (e.g., CSCWD, C\&T, UIST, IUI).

Concerning the process of citation retrieval, Google Scholar ${ }^{2}$ was chosen as the main source. According to Meho and Yang [37], it constitutes a freely available service that covers more papers when compared to subscription-based databases like Web of Science and Scopus. Bibliometric data were retrieved from a total of 1520 full papers and 193 short papers between May 282016 and July 3 2016. For each record, we collected the following metadata: ID, year, venue, title, author(s), per-author affiliation, country of author's affiliation, keywords, citation count, and alternative metrics.

Before we could analyze author and affiliation metadata, we fixed misspelled errors, typos, and duplicated records. In addition, we manually standardized the authors' information (demographics and affiliation) and keywords. A total of 3509 keywords were selected from 1329 papers (approximately $85.85 \%$ of the corpus) after applying name matching and synonyms mergence, as suggested by Liu and colleagues [35]. Once standardization had been done, we then identified 5416 authors, 703 institutions, and 40 affiliation countries crawled through demographic data. Research concepts were identified from combined corpora of the four devoted outlets, while terms were aggregated based on their similarity and word frequency. Furthermore, we analyzed alternative metrics (altmetrics) to examine the coverage and scope of $\mathrm{CSCW}$ as measured by number of downloads, readers, and social mentions.

To investigate the evolution and influence of scientific collaboration in CSCW research, we adopted Melin and Persson's [38] criteria to categorize the types of collaboration. As argued by the authors, a paper can be internally (e.g., within an university or department), nationally, and internationally coauthored. We assume that a paper is co-authored if it has more than one author. In addition, the paper is institutionally coauthored if it has more than one author address suggesting that the authors come from different institutions or research units.

The scientific impact of co-authored papers is measured by calculating the average number of citations per co-authored paper. We follow the Bu et al.'s [21] guidelines to analyze aspects such as the proportion of co-authored papers that have received at least ten citations, the average number of authors in co-authored papers, and the influence of collaboration types on the average number of citations at each venue.

\section{RESULTS AND DISCUSSION}

Scientific communities can be characterized as clusters of authors with critical roles in modern science [39]. Recent studies (e.g., [7]) indicate that the number of collaborators has increased considerably in the field of CSCW. Such results are in line with other research communities constituted by a sizeable connected component in the co-authorship network

\footnotetext{
${ }^{2}$ https://scholar.google.com
}

[40]. Repeat authorship is an important metric for measuring community forming and growth. Our results showed a total of 30 authors with 10 or more papers, 17 of which were females. The average number of authors per paper is more accentuated in the ACM CSCW conference series, followed by ECSCW, GROUP, and JCSCW. We also denote an approximate average of 3 authors per paper, and 2485 authors (45.9\%) published more than one paper. Looking at the results provided by Table I, GROUP and ACM CSCW have approximately 3 to 4 authors per paper, whilst ECSCW and JCSCW have a prevalence of 1 to 2 authors per paper [7]. We are also able to conclude that the CSCW outlets examined here have similar values in terms of mean team size (as measured by the number of authors per paper) when compared to HCI conferences.

TABLE I. COUNT OF PAPERS WITH 1-2, 3-4, 5-6, 7-8, AND >8 AUTHORS.

\begin{tabular}{|c|c|c|c|c|}
\hline \multirow{2}{*}{$\#$} & \multicolumn{4}{|c|}{ Venues } \\
\cline { 2 - 5 } & GROUP & $A C M C S C W$ & $E C S C W$ & $J C S C W$ \\
\hline 1 to 2 & 129 & 325 & 77 & 153 \\
\hline 3 to 4 & 144 & 464 & 59 & 83 \\
\hline 5 to 6 & 22 & 149 & 24 & 23 \\
\hline 7 to 8 & 2 & 40 & 1 & 5 \\
\hline$>8$ & 1 & 7 & 4 & 1 \\
\hline
\end{tabular}

A quantitative analysis of the $\mathrm{CHI}$ conference [5] identified some influential citation factors when considering the authors' affiliation institution and country. In other words, the type of collaboration has a significant impact on citations. Fig. 1 plots such collaborative interactions using Melin and Persson's [38] categories. The effect of scientific collaboration on CSCW research is primarily expressed at a local level between researchers from the same organization, followed by domestic co-authorships among researchers from different institutions within the same country, international collaborations between authors from different institutions situated in distinct countries, and individual authorships [7].

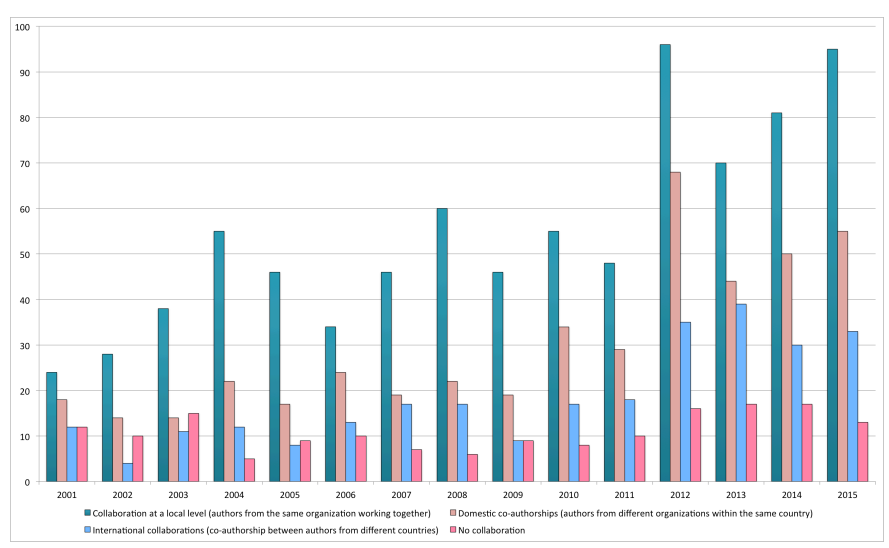

Fig. 1. Distribution of collaboration types by year.

Regarding the geographical distribution of the accumulated contribution to the CSCW literature (Fig. 2), a prevalence of institutions from USA $(54.47 \%)$ is visible for all venues. In contrast to other outlets such as CSCWD which attracts papers from predominantly East Asia (i.e., China), the chosen venues are also mainly represented by authors from institutions located in UK, Canada, Germany, Denmark, France, and Australia. 


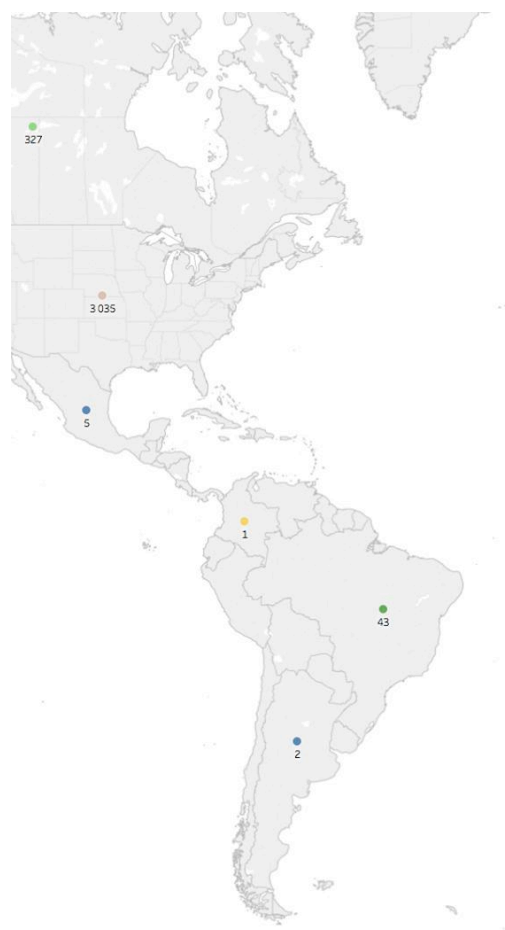

Fig. 2. Geographical distribution of the accumulated contribution.

Despite the highly interdisciplinary nature of CSCW, our study provides further evidence for situations in which research collaborations take place among organizations located in the same country with a reduced number of outside connections. According to Milard and Tanguy [28], "citations of co-workers from the same lab tend to be repeated more often". It is worth noting that most of the international collaborations in this study involve only one author from an external institution.

Concerning the type of research collaboration and its impact on the average number of citations (Fig. 3), the highest influence of internal (local) collaborations on citations is followed by domestic co-authorships and international collaborations [7]. As shown in Fig. 3, only JCSCW presented a set of individual authorships with more impact than international collaborations.

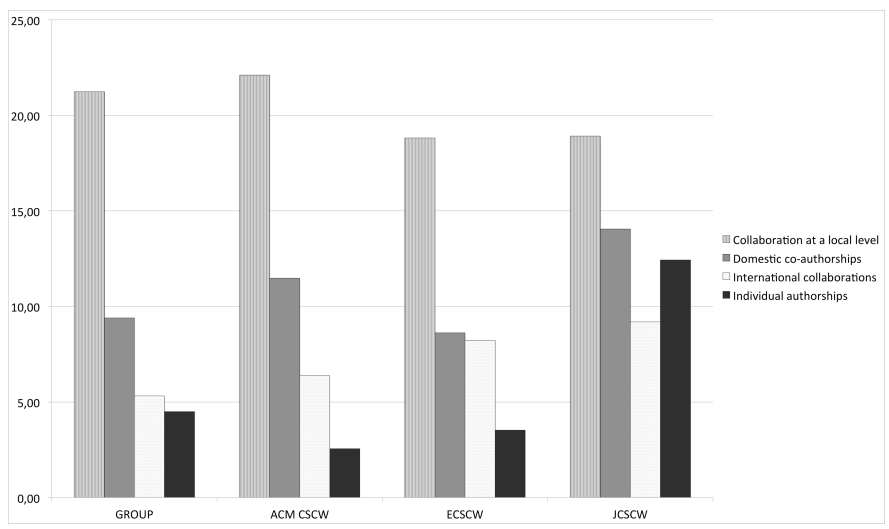

Fig. 3. Influence of collaboration types on the average citations.

Previous studies (e.g., $[4,11])$ have demonstrated that the geographical proximity of members in a research community is

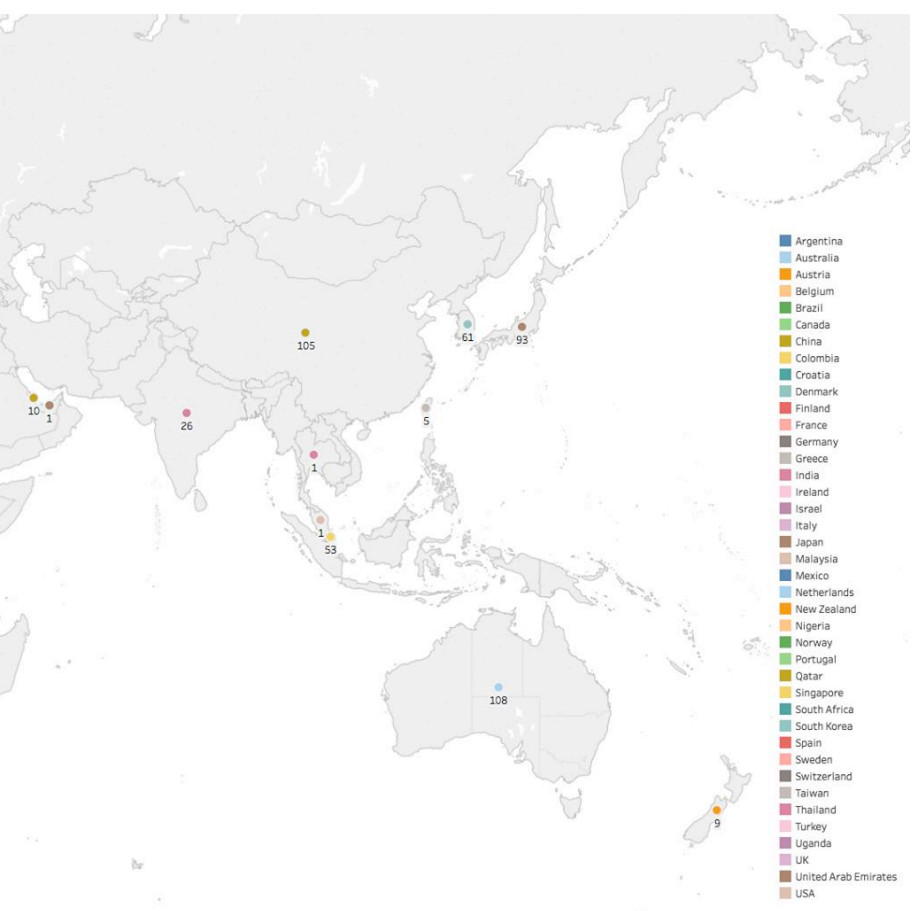

an important factor in establishing collaborations between HCI and CSCW authors. Thus, this work furthers an existing strand of research by identifying a pattern of repeat authorship and close interactions among CSCW researchers. These values correlate satisfactorily with Petersen [12] and further support the idea that collaborations involving strong ties gain $17 \%$ more citations. Our sample also presents a main authorship component constituted by authors from universities and research labs $(n=5212)$ when compared to corporate professionals $(n=170)$. This result suggests that the few interactions between academia and industry might mean that the industrial sector may not be "broad enough to satisfy the collaborative needs of the universities" [38].

The potential of future discoveries is highly constrained by the tendency of researchers to study topics already established or related to their current expertise. Fortunato and colleagues [2] go even further by arguing that researchers usually avoid the risk of failure, despite some recent evidence pointing to the higher impact of innovative publications. A keyword analysis allowed to identify the most addressed topics in our sample by frequency range (Table II). Active topics studied in CSCW papers include social media, awareness, crowdsourcing, health care, among others [7]. We identified some themes by using Jacovi et al.'s [11] methodological guidelines. Theories and models constitute the main theme in CSCW research, including terms like activity theory, ethnomethodology, social network analysis, distributed cognition, and workplace studies. CSCW is the second most representative theme, being constituted by general terms introduced or already established in the field (e.g., common ground and articulation work). An emphasis on system design and evaluation seems to be slightly replaced by studies of already deployed systems and technologies [7]. This confirms prior findings in the literature (e.g., [6]). Moreover, it should be noted that the topics addressed are intimately related to the different scope of European and North American CSCW communities. 
TABLE II. MOST FREQUENT KEYWORDS (2001-2015).

\begin{tabular}{|c|c|}
\hline \# & Level 1 \\
\hline$>=15$ & $\begin{array}{l}\text { CSCW (108), collaboration (102), ethnography (70), CMC (68), social } \\
\text { media (66), awareness (58), crowdsourcing (50), coordination (46), online } \\
\text { communities (42), health care (41), social computing (40), communication } \\
\text { (39), Wikipedia (38), privacy (36), social networks (33), social networking } \\
\text { sites (32), design (32), Facebook (31), Twitter (30), groupware (24), instant } \\
\text { messaging (23), cyberinfrastructure (23), ethnomethodology (22), } \\
\text { empirical studies (22), work practices (20), crisis informatics (18), } \\
\text { cooperative work (17), communities of practice (17), learning (17), } \\
\text { infrastructure (17), trust (17), activity theory (16), electronic mail (16), } \\
\text { knowledge management (16), evaluation (15), field studies (15), video } \\
\text { conferencing (15), community (15), HCI (15), wikis (15) }\end{array}$ \\
\hline \# & Level 2 \\
\hline $\begin{array}{l}<15 \\
\text { and } \\
>=10\end{array}$ & $\begin{array}{l}\text { social networking (14), ubiquitous computing (14), e-Science (14), } \\
\text { articulation work (13), distributed cognition (13), conversation analysis } \\
\text { (13), participatory design (13), children (13), software development (13), } \\
\text { collaborative work (12), cooperation (12), social network analysis (12), } \\
\text { CSCL (12), education (12), creativity (12), decision making (12), games } \\
\text { (12), interruptions (12), mobile (12), scientific collaboration (11), } \\
\text { consistency management (11), distributed work (11), distributed teams } \\
\text { (11), information sharing (11), participation (11), social interaction (11), } \\
\text { social capital (11), user-generated content (11), case studies (10), } \\
\text { communities (10), collaborative systems and tools (10), collaborative } \\
\text { design (10), collaborative virtual environments (10), teams (10), boundary } \\
\text { objects (10), culture (10), emotion (10), information seeking (10), } \\
\text { integration (10), motivation (10), operational transformation (10) }\end{array}$ \\
\hline \# & \\
\hline $\begin{array}{l}<10 \\
\text { and } \\
>=8\end{array}$ & $\begin{array}{l}\text { user studies (9), chat (9), blogs (9), microblogging (9), health (9), social } \\
\text { software (9), socio-technical systems (9), mobile phones (9), social search } \\
\text { (9), disasters (9), place (9), peer production (9), social support (8), video } \\
\text { (8), social awareness (8), citizen science (8), human computation (8), } \\
\text { electronic health records (8), health informatics (8), identity (8), usability } \\
\text { (8), information infrastructures (8), software engineering (8), virtual worlds } \\
\text { (8), avatars (8), groups (8), virtual teams (8), interaction (8), language (8), } \\
\text { context-aware computing (8), affect (8), co-located collaboration (8), home } \\
\text { (8), enterprise (8), family (8), recommender systems (8), sustainability (8), } \\
\text { gender (8), workplace (8), visualization (8), policy (8), rhythms (8) }\end{array}$ \\
\hline
\end{tabular}

This study has largely followed from our previous analysis in Correia and colleagues [7]. As argued by $\mathrm{Bu}$ and co-workers [21], a multivariate set of views is required to understand scientific collaboration. Combining bibliometric indicators and alternative metrics, we can draw new insights and gain a better understanding of the structure and impact of research collaboration in the field of CSCW (Table III). Observation of the authorship data shows approximately a triad (3 authors per co-authored paper) in all venues. This is consistent with $\mathrm{Bu}$ et al.'s [21] claim that researchers continually working in large teams tend to publish lower-impact publications. In addition, Fortunato and co-workers [2] also denoted that large teams tend to achieve a short-lived impact. In their analysis of team size and scientific impact in science and engineering, Larivière and co-workers [20] revealed that a team-authored paper has a greater visibility and can be 6.3 times more prone to achieve 1000 citations than a solo-authored paper. As detailed in Table III, we found a higher impact of co-authored papers (measured by the average number of citations) when compared to soloauthored papers. Furthermore, the percentage of co-authored papers that have received at least ten citations (i.e., more than $65 \%$ in all venues) has further strenghened our confidence in the influence of collaboration on CSCW.

Extrapolating to the use of alternative metrics to evaluate research outputs, our results indicate a higher number of downloads for co-authored papers in GROUP and ACM CSCW. Nevertheless, a lower impact of downloads and readers on citations per co-authored paper is visible when compared to solo-authored papers. This also true for the average number of downloads, readers, and social mentions per paper. However, given the small sample size, caution must be exercised and the need for further research in this field is imperative.

TABLE III. BIBLIOMETRICS AND ALTERNATIVE METRICS OF COLLABORATIVE COMPUTING RESEARCH BETWEEN 2001 AND 2015.

\begin{tabular}{|c|c|c|c|c|}
\hline \multirow{2}{*}{ Criteria } & \multicolumn{4}{|c|}{ Venues } \\
\cline { 2 - 5 } & GROUP & $A C M C S C W$ & $E C S C W$ & $J C S C W$ \\
\hline B1 & 298 & 985 & 165 & 265 \\
\hline B2 & 2.86 & 3.34 & 3.12 & 2.64 \\
\hline B3 & 3.08 & 3.56 & 3.28 & 3.12 \\
\hline B4 & 41.77 & 43.55 & 40.58 & 54.37 \\
\hline B5 & 33.25 & 27.16 & 22.73 & 52.93 \\
\hline B6 & $65.7 \%$ & $67.39 \%$ & $66.23 \%$ & $78.64 \%$ \\
\hline B7 & $68.75 \%$ & $58.06 \%$ & $53.33 \%$ & $81.36 \%$ \\
\hline A1 & 738.18 & 658.96 & 237.9 & ---- \\
\hline A2 & 726.94 & 513.11 & 262.2 & ---- \\
\hline A3 & ---- & ---- & 12.82 & 9.56 \\
\hline A4 & ---- & ---- & 17.13 & 16.59 \\
\hline A5 & ---- & ---- & 0.07 & 0.37 \\
\hline A6 & ---- & ---- & 0.07 & 0.39 \\
\hline A7 & 17.67 & 15.13 & 5.86 & ---- \\
\hline A8 & 21.86 & 18.89 & 11.53 & ---- \\
\hline A9 & ---- & ---- & 0.32 & 0.18 \\
\hline A10 & --- & ---- & 0.75 & 0.31 \\
\hline
\end{tabular}

a. Overall criteria of the quantitative assessment of $\mathrm{CSCW}$ research organized by: total number of papers (B1), average number of authors per paper (B2), average number of authors per co-authored paper (B3), average number of citations per co-authored paper (B4), average number of citations per soloauthored paper (B5), percentage of co-authored papers that have received at least ten citations (B6), percentage of solo-authored papers that have received at least ten citations (B7), average number of downloads per co-authored paper (A1), average number of downloads per solo-authored paper (A2), average number of readers per co-authored paper ( $\mathbf{A 3}$ ), average number of readers per solo-authored a mentions per solo-authored paper (A6), impact of downloads on citations per co-authored paper (A7), impact of downloads on citations per solo-authored paper (A8), impact of readers on citations per coauthored paper (A9), and impact of readers on citations per solo-authored paper (A10).

\section{CONCLUSION}

The initial exploration of the effects of collaboration on CSCW research over a period of 15 years has reinforced the need of retrieving evidence to reflect on the publication data and thus characterizing the field and its evolution over time. It should be noted that the techniques used in this study are not without problems, both in the data retrieval and analysis phases. Some problems involve treating short and full papers in the same way and acknowledging self-citations in the same level of relevance of external ones. Our study is also limited in terms of disciplinary coverage and sample size. If we want to understand the collaborative patterns among scholars in CSCW we need to integrate findings from multiple disciplines based on a wide range of data and methods.

Despite the encouraging findings of this work, there is still much room left for further research. Among the limitations of this work is that it only separately investigates how CSCW subdomains interact with each other. As future work we aim to examine the relationship between topics and authors. Further experimental investigations are needed to incorporate largescale analysis using computational models such as topic models [31]. For instance, an important issue to resolve for future studies is the characterization of relationships between documents, authors, topics, and words using the author-topic model [13]. Another line of potential research is mapping the context of citations in CSCW literature. Moreover, determining the reasons stimulating researchers to collaborate [34], the strength of ties in long-term co-authorships [12], and the role of collaborative stability and persistence [21] can be worth mentioning as future research directions. 


\section{ACKNOWLEDGMENTS}

This work is supported by the Portuguese Foundation for Science and Technology (Fundação para a Ciência e a Tecnologia - FCT), under the project CMU/CS/0012/2017 "eCSAAP - expert Crowdsourcing for Semantic Annotation of Atmospheric Phenomena".

\section{REFERENCES}

[1] A. L. Barabâsi, H. Jeong, Z. Néda, E. Ravasz, A. Schubert, and T. Vicsek, "Evolution of the social network of scientific collaborations," Physica A: Statistical Mechanics and its Applications, vol. 311, 3-4, pp. 590-614, 2002.

[2] S. Fortunato, C. T. Bergstrom, K. Börner, J. A. Evans, D. Helbing, S. Milojević, ... and A. Vespignani, "Science of science," Science, vol. 359, 6379, eaao0185, 2018.

[3] D. Shapiro, "The limits of ethnography: combining social sciences for CSCW," In Proceedings of the ACM Conference on Computer Supported Cooperative Work, pp. 417-428, 1994.

[4] D. B. Horn, T. A. Finholt, J. P. Birnholtz, D. Motwani, and S. Jayaraman, "Six degrees of Jonathan Grudin: a social network analysis of the evolution and impact of CSCW research. In Proc. of the ACM Conf. on Computer Supported Cooperative Work (pp. 582-591), 2004.

[5] C. Bartneck and J. Hu, "The fruits of collaboration in a multidisciplinary field," Scientometrics, vol. 85, 1, pp. 41-52, 2010.

[6] B. Keegan, D. Horn, T. A. Finholt, and J. Kaye, "Structure and dynamics of coauthorship, citation, and impact within CSCW," arXiv preprint arXiv:1307.7172, 2013.

[7] A. Correia, H. Paredes, and B. Fonseca, "Scientometric analysis of scientific publications in CSCW," Scientometrics, vol. 114, 1, pp. 31-89, 2018.

[8] J. R. Wallace, S. Oji, and C. Anslow, "Technologies, methods, and values: changes in empirical research at CSCW 1990-2015," In Proceedings of the ACM Hum.-Comput. Interact., vol. 1, 2, 106, 2017.

[9] A. Correia, H. Paredes, and B. Fonseca, "Reframing taxonomy development in collaborative computing research: a review and synthesis of CSCW literature 2003-2010," In Proceedings of Int. Conf. on Collaboration and Technology (pp. 42-59), 2018.

[10] C. W. Holsapple and W. Luo, "A citation analysis of influences on collaborative computing research," Computer Supported Cooperative Work, vol. 12, 3, pp. 351-366, 2003.

[11] M. Jacovi, V. Soroka, G. Gilboa-Freedman, S. Ur, E. Shahar, and N. Marmasse, "The chasms of CSCW: a citation graph analysis of the CSCW conference," In Proceedings of the ACM Conference on Computer Supported Cooperative Work (pp. 289-298), 2006.

[12] A. M. Petersen, "Quantifying the impact of weak, strong, and super ties in scientific careers," National Academy of Sciences, vol. 112, 34, E4671-E4680, 2015.

[13] M. Rosen-Zvi, T. Griffiths, M. Steyvers, and P. Smyth, "The authortopic model for authors and documents," In Proceedings of the Conference on Uncertainty in Artificial Intelligence (pp. 487-494), 2004.

[14] D. S. Price, "Little science, big science," New York City: Columbia University Press, 1963.

[15] V. V. Nalimov and B. M. Mulchenko, "Scientometrics. Studies of science as a process of information," Moscow: Science, 1969.

[16] L. Leydesdorff, "The challenge of scientometrics: the development, measurement, and self-organization of scientific communications," Universal-Publishers, 2001.

[17] A. Van Raan, "Scientometrics: state-of-the-art," Scientometrics, vol. 38, 1, pp. 205-218, 1997.

[18] J. Mingers and L. Leydesdorff, "A review of theory and practice in scientometrics," European Journal of Operational Research, vol. 246, 1, pp. 1-19, 2015.

[19] S. Wuchty, B. F. Jones, and B. Uzzi, "The increasing dominance of teams in production of knowledge," Science, vol. 316, 5827, pp. 1036$1039,2007$.
[20] V. Larivière, Y. Gingras, C. R. Sugimoto, and A. Tsou, "Team size matters: collaboration and scientific impact since 1900," J Assoc Inf Sci Technol., vol. 66, pp. 1323-1332, 2015.

[21] Y. Bu, D. S. Murray, Y. Ding, Y. Huang, and Y. Zhao, "Measuring the stability of scientific collaboration," Scientometrics, vol. 114, 2, pp. 463-479, 2018

[22] S. Davoudi and J. Pendlebury, "Centenary paper: the evolution of planning as an academic discipline," Town Planning Review, vol. 81, 6, pp. 613-646, 2010.

[23] J. Xu, Y. Ding, and V. Malic, "Author credit for transdisciplinary collaboration," PloS One, vol. 10, 9, e0137968, 2015.

[24] B. Uzzi, S. Mukherjee, M. Stringer, and B. Jones, "Atypical combinations and scientific impact," Science, vol. 342, 6157, pp. 468472, 2013.

[25] Y. Bu, Y. Ding, J. Xu, X. Liang, G. Gao, and Y. Zhao, "Understanding success through the diversity of collaborators and the milestone of career," Journal of the Association for Information Science and Technology, vol. 69, 1, pp. 87-97, 2018.

[26] W. Glänzel and A. Schubert, "Analysing scientific networks through coauthorship," In Handbook of Quantitative Science and Technology Research, pp. 257-276, 2004

[27] T. Amjad, Y. Ding, J. Xu, C. Zhang, A. Daud, J. Tang, and M. Song, "Standing on the shoulders of giants," Journal of Informetrics, vol. 11, 1, pp. 307-323, 2017.

[28] B. Milard and L. Tanguy, "Citations in scientific texts: do social relations matter?," Journal of the Association for Information Science and Technology, vol. 69, 11, pp. 1380-1395, 2018.

[29] K. Crowston, E. Mitchell, and C. Østerlund, "Coordinating advanced crowd work: extending citizen science," In Proceedings of the Hawaii International Conference on System Sciences (pp. 1681-1690), 2018.

[30] S. Schaltegger, M. Beckmann, and E. G. Hansen, "Transdisciplinarity in corporate sustainability: mapping the field," Business Strategy and the Environment, vol. 22, 4, pp. 219-229, 2013.

[31] D. Hall, D. Jurafsky, and C. D. Manning, "Studying the history of ideas using topic models," In Proceedings of the Conference on Empirical Methods in Natural Language Processing (pp. 363-371), 2008.

[32] G. Convertino, T. G. Kannampallil, and I. Councill, "Mapping the intellectual landscape of CSCW research," Posters on ACM Conference on Computer Supported Cooperative Work, 6, 2006.

[33] J. Wainer and C. Barsottini, "Empirical research in CSCW - a review of the ACM/CSCW conferences from 1998 to 2004," Journal of the Brazilian Computer Society, vol. 13, 3, pp. 27-35, 2007.

[34] H. Iglič, P. Doreian, L. Kronegger, and A. Ferligoj, "With whom do researchers collaborate and why?," Scientometrics, vol. 112, 1, pp. 153174, 2017.

[35] Y. Liu, J. Goncalves, D. Ferreira, B. Xiao, S. Hosio, and V. Kostakos, "CHI 1994-2013: mapping two decades of intellectual progress through co-word analysis," In Proceedings of the ACM Conference on Human Factors in Computing Systems (pp. 3553-3562), 2014.

[36] O. Mubin, A. Al Mahmud, and M. Ahmad, "HCI down under: reflecting on a decade of the OzCHI conference," Scientometrics, vol. 112, 1, pp. 367-382, 2017.

[37] L. I. Meho and K. Yang, "Impact of data sources on citation counts and rankings of LIS faculty: Web of Science versus Scopus and Google Scholar," J Am Soc Inf Sci Technol., vol. 58, 13, pp. 2105-2125, 2007.

[38] G. Melin and O. Persson, "Studying research collaboration using coauthorships," Scientometrics, vol. 36, 3, pp. 363-377, 1996.

[39] J. Mao, Y. Cao, K. Lu, and G. Li, "Topic scientific community in science: a combined perspective of scientific collaboration and topics," Scientometrics, vol. 112, 2, pp. 851-875, 2017.

[40] L. M. Bettencourt, D. I. Kaiser, and J. Kaur, "Scientific discovery and topological transitions in collaboration networks," Journal of Informetrics, vol. 3, 3, pp. 210-221, 2009.

[41] A. Correia, D. Schneider, B. Fonseca, and H. Paredes, "Crowdsourcing and massively collaborative science: a systematic literature review and mapping study," In Proceedings of the International Conference on Collaboration and Technology (pp. 133-154), 2018. 